\title{
ON TWO CIRCULAR INCLUSIONS IN HARMONIC PROBLEMS
}

\author{
BY \\ E. HONEIN, T. HONEIN, AND G. HERRMANN \\ Stanford University, Stanford, California
}

\begin{abstract}
In this paper, we derive the solution for two circular cylindrical elastic inclusions perfectly bonded to an elastic matrix of infinite extent, under anti-plane deformation. The two inclusions have different radii and possess different elastic properties. The matrix is subjected to arbitrary loading. The solution is obtained, via iterations of Möbius transformations, as a rapidly convergent series with an explicit general term involving the complex potential of the corresponding homogeneous problem, i.e., when the inclusions are absent and the matrix material occupies the entire space and is subjected to the same loading. This procedure has been termed "heterogenization."

The technique used can be applied to problems governed by Laplace's equation.

Finally some remarks are included concerning the relation of our solution to the theory of discontinuous groups and automorphic functions and possible generalizations to multiple inclusions.
\end{abstract}

1. Introduction. Some time ago, Milne-Thomson advanced the circle theorem in fluid mechanics [1, 2]. It relates the solution of potential flow around a rigid circular cylinder to that of the potential flow without the cylinder and driven by the same sources. This theorem proved to be very useful in some applications in aero- and hydrodynamics. It is to be noted that the counterpart of this theorem in electrostatics is known as the "method of inversion" (see, e.g., [3] or [4]) and goes back to the work of Lord Kelvin.

More recent work by T. Honein and G. Herrmann extends this theorem to thermoelasticity and elasticity [5-8]. In particular, the problem of an elastic body containing an elastic circular inclusion and subject to arbitrary loading is treated in a unified and concise manner, in terms of the corresponding homogeneous problem (heterogenization).

The purpose of this study is to advance and apply a two-circular-inclusion theorem in field theories governed by Laplace's equation. These include, for example, potential fluid flow, electrostatics, membrane theory, and anti-plane elasticity. We choose the latter in order to be specific in carrying out the following developments. 
The theorem relates the solution, of a medium containing two elastic circular cylindrical inclusions, either outside of each other, or one inside the other, to that of a homogeneous medium, via iterations of Möbius transformations [9], enabling one to write down the solution as a rapidly convergent series. The interesting special case when the circles are tangent to each other is also discussed.

2. Anti-plane formulation of elastostatics. The purpose of this section is to recall the basic formulation of anti-plane elastostatics and to establish some notations that will be used throughout the paper.

A material body is said to undergo anti-plane deformation when the displacement field satisfies

$$
\begin{gathered}
u_{1}=u_{2}=0, \\
u_{3}=u_{3}\left(x_{1}, x_{2}\right),
\end{gathered}
$$

i.e., the only nonvanishing component of displacement, with respect to a Cartesian coordinate system $O x_{1} x_{2} x_{3}$, is $u_{3}$, which is a function of the coordinates $x_{1}$ and $x_{2}$ only.

The only nonvanishing infinitesimal strain components are

$$
\begin{gathered}
\varepsilon_{13}=\varepsilon_{31}=\frac{1}{2} \frac{\partial u_{3}}{\partial x_{1}}, \\
\varepsilon_{23}=\varepsilon_{32}=\frac{1}{2} \frac{\partial u_{3}}{\partial x_{2}} .
\end{gathered}
$$

For a linear (homogeneous and isotropic) elastic body, the only nonvanishing stress components are

$$
\begin{aligned}
& \sigma_{13}=\sigma_{31}=\mu \frac{\partial u_{3}}{\partial x_{1}}, \\
& \sigma_{23}=\sigma_{32}=\mu \frac{\partial u_{3}}{\partial x_{2}},
\end{aligned}
$$

where $\mu$ is the shear modulus.

If we assume zero body forces, the equilibrium equations

$$
\sigma_{i j, j}=0
$$

require

$$
\nabla^{2} u_{3}=0
$$

Let $u$ denote $u_{3}, u$ being a harmonic function; it may be considered as the imaginary part of a holomorphic function $\phi$ scaled by a factor $1 / \mu$; i.e.,

$$
u=\frac{1}{\mu} \operatorname{Im}\{\phi(z)\}=\frac{1}{\mu} \frac{\phi-\bar{\phi}}{2 i}
$$

where $z=x_{1}+i x_{2}, i$ the imaginary unit, and the bar indicates complex conjugation. 
From (9) it can easily be checked that

$$
\begin{gathered}
\frac{\partial u}{\partial x_{1}}=\frac{1}{\mu} \operatorname{Im}\left\{\phi^{\prime}\right\}, \\
\frac{\partial u}{\partial x_{2}}=\frac{1}{\mu} \operatorname{Re} \phi^{\prime}=\frac{1}{\mu} \frac{\phi^{\prime}+\overline{\phi^{\prime}}}{2},
\end{gathered}
$$

where prime indicates differentiation with respect to the argument.

The stress components as a function of $\phi$ read

$$
\begin{aligned}
& \sigma_{13}=\operatorname{Im} \phi^{\prime}, \\
& \sigma_{23}=\operatorname{Re} \phi^{\prime},
\end{aligned}
$$

which are equivalent to the single complex equation

$$
\sigma_{23}+i \sigma_{13}=\phi^{\prime} \text {. }
$$

The stress components in polar coordinates are

$$
\sigma_{z \theta}+i \sigma_{z r}=e^{i \theta} \phi^{\prime}
$$

as can readily be verified.

3. Solution of the anti-plane problem in the presence of one circular inclusion. In this section, we consider the heterogeneous problem of an elastic circular inclusion perfectly bonded to an elastic matrix of infinite extent. The matrix is subjected to arbitrary singularities (loads) that produce an anti-plane deformation, but are otherwise arbitrary (see Fig. 1). It is the purpose of this section to recall briefly how the solution of this problem can be obtained by simple algebraic manipulations from the solution of the corresponding homogeneous problem, i.e., when the full space is occupied by the matrix material and subjected to the same singularities. This method can be regarded, in the present context, as an extension of the Milne-Thomson circle theorem, and was applied by Smith [10] and independently by Lin, Honein, and Herrmann [11] to problems in anti-plane elasticity.

Assume that $\phi$, the complex potential introduced in the previous section, represents the solution of an anti-plane problem of an infinite body. In a region free of

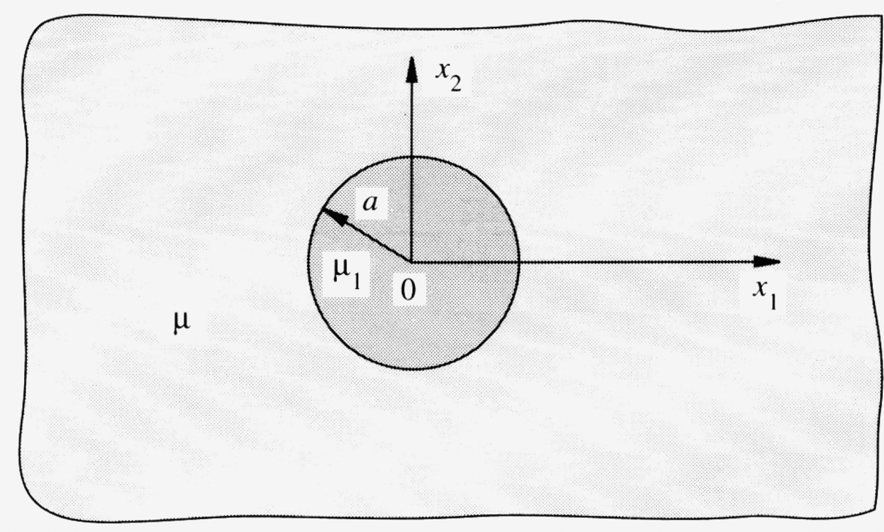

FIG. 1. One circular inclusion in an infinite medium. 
singularities a circular elastic inclusion of radius $a$ is introduced. The most general complex potentials for this heterogeneous problem are sought in the form:

inside the inclusion:

$$
\phi+g
$$

outside the inclusion:

$$
\phi+\hat{f},
$$

where $g$ and $f$ are holomorphic functions inside the disk $r<a$ and yet to be determined. The origin of our coordinate system is taken to be the center of the inclusion.

The "hat" transformation is defined by

$$
\hat{f}(z)=\overline{f\left(a^{2} / \bar{z}\right)}
$$

and maps analytic functions from inside the disk $r<a$ to its outside including the point at infinity and vice versa.

Under this form for the solution no new singularities are introduced, and the problem reduces to finding $g$ and $f$ such that the boundary conditions are satisfied. To this end, we designate by $\mu_{1}$ and $\mu$ the shear moduli of the inclusion and the matrix, respectively.

The continuity of traction, along the circular interface requires

$$
\operatorname{Im}\left\{e^{i \theta}(\phi+g)^{\prime}\right\}=\operatorname{Im}\left\{e^{i \theta}(\phi+\hat{f})^{\prime}\right\} \quad \text { along } z=a e^{i \theta} .
$$

This yields

$$
\operatorname{Im}\left\{\frac{z}{a} g^{\prime}\right\}=\operatorname{Im}\left\{-\frac{a}{z} \overline{f^{\prime}(z)}\right\}=\operatorname{Im}\left\{\frac{z}{a} f^{\prime}(z)\right\},
$$

which is satisfied for

$$
f=g \text {. }
$$

The continuity of displacement, along the circular interface, requires

$$
\frac{1}{\mu_{1}} \operatorname{Im}(\phi+g)=\frac{1}{\mu} \operatorname{Im}(\phi+\hat{f})=\frac{1}{\mu} \operatorname{Im}(\phi+\bar{f})=\frac{1}{\mu} \operatorname{Im}(\phi+\bar{g})=\frac{1}{\mu} \operatorname{Im}(\phi-g),
$$

which is satisfied for

$$
\frac{1}{\mu_{1}}(\phi+g)=\frac{1}{\mu}(\phi-g),
$$

i.e., for

$$
g=\frac{\mu_{1}-\mu}{\mu_{1}+\mu} \phi=\alpha \phi
$$

where

$$
\alpha=\left(\mu_{1}-\mu\right) /\left(\mu_{1}+\mu\right) .
$$

In summary, the solution reads

inside the inclusion:

$$
\phi+g=(1+\alpha) \phi,
$$

inside the matrix:

$$
\phi+\hat{f}=\phi+\alpha \hat{\phi} .
$$


The previous solution was given when the origin coincided with the center of the inclusion. For an inclusion of radius $a$ centered at $z_{0}$, the solution must read inside the inclusion:

inside the matrix:

$$
\phi+g=(1+\alpha) \phi,
$$

$$
\phi+\alpha \phi \overline{\left(\frac{a^{2}}{\bar{z}-\bar{z}_{0}}+z_{0}\right)} .
$$

4. Solution of the anti-plane problem in the presence of two circular inclusions of different materials and different radii. In this section, we use the solution presented in the previous section as a building block to construct the solution of two circular inclusions of radii $a_{1}, a_{2}$ and shear moduli $\mu_{1}$ and $\mu_{2}$, respectively. These inclusions are perfectly bonded to a matrix of shear modulus $\mu$ that is subjected to arbitrary singularities (see Fig. 2). The method to be presented can be regarded as an extension of the Schwarz alternating method, which in principle permits one to obtain the solution to any degree of accuracy. However, here the analysis will be carried a step further. By exploiting the Möbius transformation, the general term of the series is obtained and, therefore, the general solution is written as a rapidly convergent series with an explicit general term.

To this end we define

$$
A_{i} z=a_{i}^{2} /\left(\bar{z}-\bar{z}_{i}\right)+z_{i}, \quad i=1,2,
$$

where $z_{1}, z_{2}$ are the centers of the inclusions, and

$$
\alpha_{i}=\left(\mu_{i}-\mu\right) /\left(\mu_{i}+\mu\right), \quad i=1,2 .
$$

The derivation of the solution proceeds as follows:

1. Consider the solution of the region without inclusions (i.e., $\phi(z))$.

2. Modify the solution taking into account the presence of the first inclusion. Then the modified solution is

inside the first inclusion:

$$
\phi(z)+\alpha_{1} \phi(z)
$$

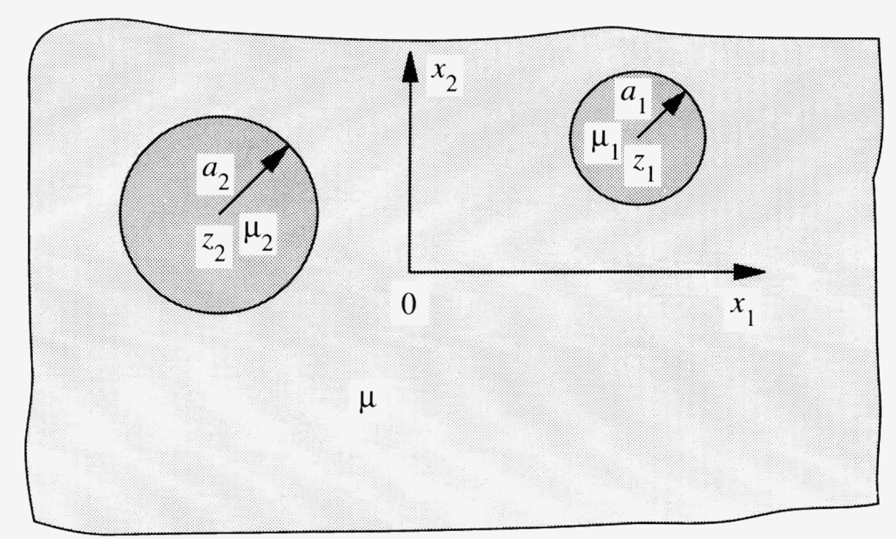

FIG. 2. Two circular inclusions, outside of each other, of arbitrary radii and arbitrary positions. 
inside the second inclusion:

$$
\phi(z)+\alpha_{1} \overline{\phi\left(A_{1} z\right)},
$$

inside the matrix:

$$
\phi(z)+\alpha_{1} \overline{\phi\left(A_{1} z\right)} .
$$

After this step, the boundary conditions are satisfied at the interface of the first inclusion but not at the interface of the second.

3. Modify the solution taking into account the presence of the second inclusion. We obtain

inside the first inclusion:

$$
\phi(z)+\alpha_{1} \phi(z)+\alpha_{2} \overline{\phi\left(A_{2} z\right)}+\alpha_{2} \alpha_{1} \phi\left(A_{1} A_{2} z\right),
$$

inside the second inclusion:

$$
\phi(z)+\alpha_{1} \overline{\phi\left(A_{1} z\right)}+\alpha_{2} \phi(z)+\alpha_{2} \alpha_{1} \overline{\phi\left(A_{1} z\right)},
$$

inside the matrix:

$$
\phi(z)+\alpha_{1} \overline{\phi\left(A_{1} z\right)}+\alpha_{2} \overline{\phi\left(A_{2} z\right)}+\alpha_{2} \alpha_{1} \phi\left(A_{1} A_{2} z\right) .
$$

After this step, the boundary conditions are satisfied at the interface of the second inclusion but not at the interface of the first.

4. Repeat steps 2 and 3 using the two additional terms each time, ad infinitum.

The net result is the following solution given as

inside the matrix:

$$
\begin{aligned}
\phi(z) & +\alpha_{1} \overline{\phi\left(A_{1} z\right)}+\alpha_{2} \overline{\phi\left(A_{2} z\right)}+\alpha_{1} \alpha_{2} \phi\left(A_{1} A_{2} z\right) \\
& +\alpha_{1} \alpha_{2} \phi\left(A_{2} A_{1} z\right)+\alpha_{1}^{2} \alpha_{2} \overline{\phi\left(A_{1} A_{2} A_{1} z\right)}+\cdots,
\end{aligned}
$$

which can be arranged as follows:

$$
\begin{aligned}
\phi(z) & +\alpha_{1} \alpha_{2} \phi\left(A_{1} A_{2} z\right)+\alpha_{1}^{2} \alpha_{2}^{2} \phi\left(A_{1} A_{2} A_{1} A_{2} z\right)+\cdots \\
& +\alpha_{2} \alpha_{1} \phi\left(A_{2} A_{1} z\right)+\alpha_{2}^{2} \alpha_{1}^{2} \phi\left(A_{2} A_{1} A_{2} A_{1} z\right)+\cdots \\
& +\alpha_{1} \overline{\phi\left(A_{1} z\right)}+\alpha_{1}^{2} \alpha_{2} \overline{\phi\left(A_{1} A_{2} A_{1} z\right)}+\cdots \\
& +\alpha_{2} \overline{\phi\left(A_{2} z\right)}+\alpha_{2}^{2} \alpha_{1} \overline{\phi\left(A_{2} A_{1} A_{2} z\right)}+\cdots .
\end{aligned}
$$

With $M z=A_{1} A_{2} z$ and $N z=A_{2} A_{1} z$ the solution reads

in the matrix:

$$
\begin{aligned}
\phi(z) & +\sum_{n=1}^{+\infty}\left(\alpha_{1} \alpha_{2}\right)^{n} \phi\left(M^{n} z\right)+\sum_{n=1}^{+\infty}\left(\alpha_{1} \alpha_{2}\right)^{n} \phi\left(N^{n} z\right) \\
& +\alpha_{1} \sum_{n=0}^{+\infty}\left(\alpha_{1} \alpha_{2}\right)^{n} \overline{\phi\left(A_{1} N^{n} z\right)}+\alpha_{2} \sum_{n=0}^{+\infty}\left(\alpha_{1} \alpha_{2}\right)^{n} \overline{\phi\left(A_{2} M^{n} z\right)},
\end{aligned}
$$

inside the first inclusion:

$$
\left.\left(1+\alpha_{1}\right)\left\{\phi(z)+\alpha_{2} \sum_{n=0}^{+\infty}\left(\alpha_{1} \alpha_{2}\right)^{n} \overline{\phi\left(A_{2} M^{n}\right.} \bar{z}\right)+\sum_{n=1}^{+\infty}\left(\alpha_{1} \alpha_{2}\right)^{n} \phi\left(M^{n} z\right)\right\},
$$


inside the second inclusion:

$$
\left(1+\alpha_{2}\right)\left\{\phi(z)+\alpha_{1} \sum_{n=0}^{+\infty}\left(\alpha_{1} \alpha_{2}\right)^{n} \overline{\phi\left(A_{1} N^{n} z\right)}+\sum_{n=1}^{+\infty}\left(\alpha_{1} \alpha_{2}\right)^{n} \phi\left(N^{n} z\right)\right\} .
$$

To obtain the solution we still have to determine $M^{n} z, N^{n} z, A_{1} N^{n} z$, and $A_{2} M^{n} z$. The crucial step in the following development hinges on the observation that $A_{i}(i=1,2)$ are involutary antihomographic transformations [12]. This implies that $M$ and $N=M^{-1}$ are Möbius transformations. Properties of these are exploited later to calculate efficiently the iterates $M^{n}, N^{n}, A_{2} M^{n}$, and $A_{1} N^{n}$ and therefore the general term of the solution.

It can be readily verified from (30) that $M$ takes the form

$$
M z=A_{1} A_{2} z=(A z+B) /(C z+D)
$$

where

$$
\begin{gathered}
A=a_{1}^{2}-\left|z_{1}\right|^{2}+z_{1} \bar{z}_{2}, \\
B=\left(a_{2}^{2}-\left|z_{2}\right|^{2}\right) z_{1}-\left(a_{1}^{2}-\left|z_{1}\right|^{2}\right) z_{2}, \\
C=\bar{z}_{2}-\bar{z}_{1}, \\
D=a_{2}^{2}-\left|z_{2}\right|^{2}+\bar{z}_{1} z_{2} .
\end{gathered}
$$

Here $|\omega|$ designates the modulus of the complex number $\omega$.

The transformation $\mathrm{Nz}$ is obtained from the previous expression by merely interchanging the indices 1 and 2 .

The next step is to write $M$ and $N$ in normal form [12]. To this end, we distinguish two cases:

CASE 1. $M$ (or $N$ ) has two distinct fixed points.

In this case $M$ can be given by

$$
\frac{M z-\gamma_{1}}{M z-\gamma_{2}}=k \frac{z-\gamma_{1}}{z-\gamma_{2}} .
$$

A straightforward calculation leads to

$$
\begin{aligned}
& \gamma_{1}=\frac{L+\sqrt{\Delta}}{2 C}, \\
& \gamma_{2}=\frac{L-\sqrt{\Delta}}{2 C},
\end{aligned}
$$

where

$$
\begin{gathered}
L=A-D=a_{1}^{2}-a_{2}^{2}+\left(z_{2}+z_{1}\right)\left(\bar{z}_{2}-\bar{z}_{1}\right), \\
\Delta=\left(a_{1}^{2}+a_{2}^{2}-\left|z_{2}-z_{1}\right|^{2}\right)^{2}-4 a_{1}^{2} a_{2}^{2}, \\
k=\frac{A+d-\sqrt{\Delta}}{A+D+\sqrt{\Delta}} .
\end{gathered}
$$

Thus $N$, which is the inverse of $M$, can be given by

$$
\frac{N z-\gamma_{1}}{N z-\gamma_{2}}=\frac{1}{k} \frac{z-\gamma_{1}}{z-\gamma_{2}} .
$$


The aforementioned formulas lead to

$$
\frac{M^{n} z-\gamma_{1}}{M^{n} z-\gamma_{2}}=k^{n} \frac{z-\gamma_{1}}{z-\gamma_{2}}
$$

and

$$
\frac{N^{n} z-\gamma_{1}}{N^{n} z-\gamma_{2}}=\frac{1}{k^{n}} \frac{z-\gamma_{1}}{z-\gamma_{2}}
$$

and, therefore, we obtain

$$
M^{n} z=\frac{\left(\gamma_{2} k^{n}-\gamma_{1}\right) z+\gamma_{1} \gamma_{2}\left(1-k^{n}\right)}{\left(k^{n}-1\right) z+\gamma_{2}-k^{n} \gamma_{1}}
$$

and

$$
N^{n} z=\frac{\left(\gamma_{1} k^{n}-\gamma_{2}\right) z+\gamma_{1} \gamma_{2}\left(1-k^{n}\right)}{\left(k^{n}-1\right) z+\gamma_{1}-k^{n} \gamma_{2}} .
$$

The formula for $A_{2} M^{n} z$ is obtained as

$$
A_{2} M^{n} z=\text { Num } / \text { Den }
$$

where

$$
\begin{aligned}
\text { Num }= & \left\{\left(a_{2}^{2}-\left|z_{2}\right|^{2}\right)\left(\overline{k^{n}-1}\right)+z_{2}\left(\overline{\gamma_{2} k^{n}-\gamma_{1}}\right)\right\} \bar{z} \\
& +\left(a_{2}^{2}-\left|z_{2}\right|^{2}\right)\left(\overline{\gamma_{2}-\gamma_{1} k^{n}}\right)+z_{2} \overline{\gamma_{1} \gamma_{2}\left(1-k^{n}\right)}
\end{aligned}
$$

and

$$
\text { Den }=\overline{\left\{\gamma_{2} k^{n}-\gamma_{1}-z_{2}\left(k^{n}-1\right)\right\} z+\gamma_{1} \gamma_{2}\left(1-k^{n}\right)-z_{2}\left(\gamma_{2}-k^{n} \gamma_{1}\right)} .
$$

The formula for $A_{1} N^{n} z$ is obtained from that for $A_{2} M^{n} z$ by simply interchanging the indices 1 and 2 .

This concludes the calculation of the general term of the solution when $\gamma_{1} \neq \gamma_{2}$.

CASE 2. $M$ (or $N$ ) has a double fixed point.

This case occurs when $\Delta=0$, i.e., when the inclusions are tangent to each other. In this case the normal form is sought as

$$
\frac{1}{M z-\gamma}=\frac{h}{z-\gamma}+l
$$

which leads to

$$
\begin{gathered}
h=1, \\
\gamma=\frac{a_{1}^{2}-a_{2}^{2}+\left(z_{1}+z_{2}\right)\left(\bar{z}_{2}-\bar{z}_{1}\right)}{2\left(\bar{z}_{2}-\bar{z}_{1}\right)}, \\
l=\frac{\bar{z}_{1}-\bar{z}_{2}}{a_{1} a_{2}} .
\end{gathered}
$$

It can be checked that $N$ is given by

$$
\frac{1}{N z-\gamma}=\frac{1}{z-\gamma}-l
$$


From the expression (62), we obtain by iteration

which leads to

$$
\frac{1}{M^{n} z-\gamma}=\frac{1}{z-\gamma}+n l
$$

Similarly

$$
M^{n} z=\frac{(1+\gamma n l) z-n l \gamma^{2}}{n l z-n l \gamma+1}
$$

$$
N^{n} z=\frac{(1-\gamma n l) z+n l \gamma^{2}}{-n l z+n l \gamma+1}
$$

Now the expressions for $A_{2} M^{n}$ and $A_{1} N^{n}$ are obtained as

$$
\begin{aligned}
& A_{2} M^{n} z=\frac{\left\{\left(a_{2}^{2}-\left|z_{2}\right|^{2}\right) \overline{n l}+z_{2}(\overline{1+n l \gamma})\right\} \bar{z}+\left(a_{2}^{2}-\left|z_{2}\right|^{2}\right)(\overline{1-n l \gamma})-z_{2} \overline{n l \gamma^{2}}}{\left(1+\overline{n l \gamma-n l z_{2}}\right) \bar{z}-\overline{n l \gamma^{2}}+\overline{z_{2}(n l \gamma-1)}}, \\
& A_{1} N^{n} z=\frac{\left\{\left(a_{1}^{2}-\left|z_{1}\right|^{2}\right) \overline{n l}+z_{1}(\overline{n l \gamma-1})\right\} \bar{z}+\left(a_{1}^{2}-\left|z_{1}\right|^{2}\right)(-1-\overline{n l \gamma})-z_{1} \overline{n l \gamma^{2}}}{\left(-1+\overline{n l \gamma-n l z_{1}}\right) \bar{z}-\overline{n l \gamma^{2}}+\overline{z_{1}(1+n l \gamma)}} .
\end{aligned}
$$

This concludes the calculation of the general term when the circular inclusions are tangent to each other.

5. Remarks concerning the applicability of the solution and convergence. In this section, we would like to make some remarks concerning the applicability of the solution derived above and the convergence of the series representation.

For the case of two circular inclusions, the solution derived in the previous section is valid when the two circular inclusions are outside of each other. However, a similar derivation can be carried out when one inclusion is completely inside the other (Fig. $3)$.

The formal series derived in the previous section, namely, equations (40)-(42), is uniformly convergent on compact sets provided $\left|\alpha_{1} \alpha_{2}\right|<1$. The case $\alpha_{i}=1$ corresponds to a rigid inclusion while $\alpha_{i}=-1$ corresponds to a circular hole. In all other cases $\left|\alpha_{i}\right|<1$.

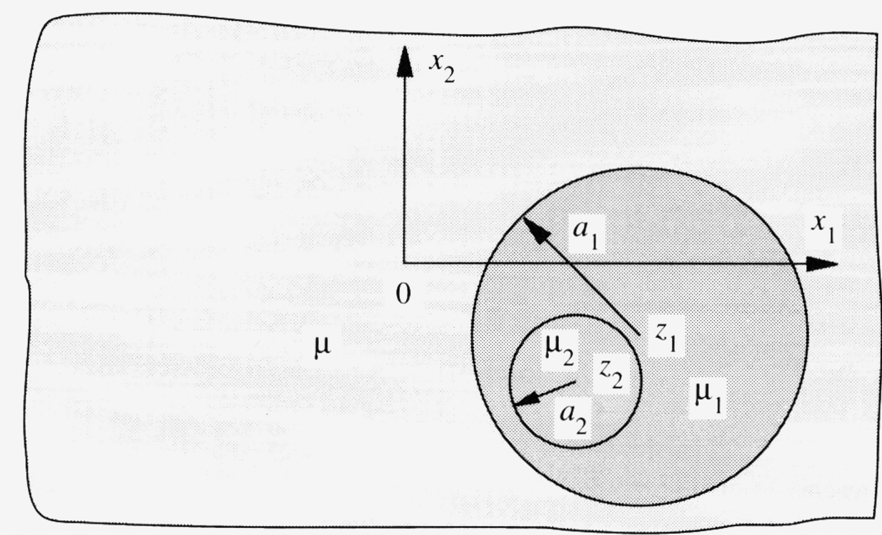

FIG. 3. Two circular inclusions, one inside the other, of arbitrary radii and arbitrary positions. 
Even for the cases of circular holes or rigid inclusions and elastic inclusions touching each other, the solution can still be obtained by differentiating the formal series solution term by term. This leads to a convergent series representing the derivative of the complex potentials of the elastic field, i.e., the expressions directly related to the stress field. To be more specific, we evaluate the derivatives involved and obtain

$$
\begin{gathered}
\left(M^{n} z\right)^{\prime}=\frac{k^{n}\left(\gamma_{1}-\gamma_{2}\right)^{2}}{\left(\left(k^{n}-1\right) z+\gamma_{2}-k^{n} \gamma_{1}\right)^{2}}, \\
\left(N^{n} z\right)^{\prime}=\frac{k^{n}\left(\gamma_{1}-\gamma_{2}\right)^{2}}{\left(\left(k^{n}-1\right) z+\gamma_{1}-k^{n} \gamma_{2}\right)^{2}}, \\
\left(\overline{\left.A_{2} M^{n} z\right)^{\prime}=\frac{-a_{2}^{2} k^{n}\left(\gamma_{1}-\gamma_{2}\right)^{2}}{\left\{\left(-\gamma_{1}+k^{n} \gamma_{2}-z_{2}\left(k^{n}-1\right)\right) z+\gamma_{1} \gamma_{2}\left(1-k^{n}\right)-z_{2}\left(\gamma_{2}-\gamma_{1} k^{n}\right)\right\}^{2}}}\right. \\
\left(\overline{A_{1} N^{n} z}\right)^{\prime}=\frac{-a_{1}^{2} k^{n}\left(\gamma_{1}-\gamma_{2}\right)^{2}}{\left\{\left(-\gamma_{2}+k^{n} \gamma_{1}-z_{1}\left(k^{n}-1\right)\right) z+\gamma_{1} \gamma_{2}\left(1-k^{n}\right)-z_{1}\left(\gamma_{1}-\gamma_{2} k^{n}\right)\right\}} .
\end{gathered}
$$

One sees that for $n$ sufficiently large, these derivatives are asymptotically proportional to $k^{n}$ if $|k|<1$, or $k^{-n}$ if $|k|>1$, and $\phi^{\prime}$ is bounded in the disk; therefore, the series will converge geometrically if the inclusions are not touching each other $(|k| \neq 1)$.

When they touch each other the derivatives are given by

$$
\begin{gathered}
\left(M^{n} z\right)^{\prime}=\frac{1}{\{1+n l(z-\gamma)\}^{2}}, \\
\left(N^{n} z\right)^{\prime}=\frac{1}{\{1-n l(z-\gamma)\}^{2}}, \\
\left(\overline{\left.A_{2} M^{n} z\right)^{\prime}=} \frac{-a_{2}^{2}}{\left\{\left(1+\gamma n l-z_{2} n l\right) z-n l \gamma^{2}+z_{2}(n l \gamma-1)\right\}^{2}},\right. \\
\left(\overline{\left.A_{1} N^{n} z\right)^{\prime}}=\frac{-a_{1}^{2}}{\left\{\left(1-\gamma n l+z_{1} n l\right) z+n l \gamma^{2}-z_{1}(n l \gamma+1)\right\}^{2}},\right.
\end{gathered}
$$

and the general term behaves proportionally to $1 / n^{2}$ for $n$ sufficiently large.

\section{Examples.}

EXAMPLE 1. Two circular inclusions disturbing a uniform shear stress.

As our first example, we consider two circular inclusions disturbing a uniform shear stress $\tau$ having complex potential $\phi(z)=\tau z$, where $\tau$ is a real constant.

A direct application of the formulae given in Secs. 4 and 5 yields the solution as 
in the matrix:

$$
\begin{aligned}
\phi_{M}^{\prime}(z)= & \tau\left(\gamma_{1}-\gamma_{2}\right)^{2} \\
\times & \left\{\sum_{n=-\infty}^{+\infty} \frac{\left(\alpha_{1} \alpha_{2}\right)^{|n|} k^{n}}{\left[\left(k^{n}-1\right) z+\gamma_{2}-k^{n} \gamma_{1}\right]^{2}}\right. \\
& -\alpha_{1} \sum_{n=0}^{+\infty} \frac{a_{1}^{2}\left(\alpha_{1} \alpha_{2} k\right)^{n}}{\left\{\left[-\gamma_{2}+k^{n} \gamma_{1}-z_{1}\left(k^{n}-1\right)\right] z+\gamma_{1} \gamma_{2}\left(1-k^{n}\right)-z_{1}\left(\gamma_{1}-\gamma_{2} k^{n}\right)\right\}^{2}} \\
& \left.-\alpha_{2} \sum_{n=0}^{+\infty} \frac{a_{2}^{2}\left(\alpha_{1} \alpha_{2} k\right)^{n}}{\left\{\left[-\gamma_{1}+k^{n} \gamma_{2}-z_{2}\left(k^{n}-1\right)\right] z+\gamma_{1} \gamma_{2}\left(1-k^{n}\right)-z_{2}\left(\gamma_{2}-\gamma_{1} k^{n}\right)\right\}^{2}}\right\},
\end{aligned}
$$

inside the first inclusion:

$$
\begin{aligned}
\phi_{I 1}^{\prime}(z)= & \left(1+\alpha_{1}\right) \tau \\
\times & \left\{1+\sum_{n=1}^{+\infty} \frac{\left(\alpha_{1} \alpha_{2} k\right)^{n}\left(\gamma_{1}-\gamma_{2}\right)^{2}}{\left[\left(k^{n}-1\right) z+\gamma_{2}-k^{n} \gamma_{1}\right]^{2}}\right. \\
& \left.\quad-\alpha_{2} \sum_{n=0}^{+\infty} \frac{a_{2}^{2}\left(\alpha_{1} \alpha_{2} k\right)^{n}\left(\gamma_{1}-\gamma_{2}\right)^{2}}{\left\{\left[-\gamma_{1}+k^{n} \gamma_{2}-z_{2}\left(k^{n}-1\right)\right] z+\gamma_{1} \gamma_{2}\left(1-k^{n}\right)-z_{2}\left(\gamma_{2}-\gamma_{1} k^{n}\right)\right\}^{2}}\right\},
\end{aligned}
$$

inside the second inclusion:

$$
\begin{aligned}
\phi_{I 2}^{\prime}(z)= & \left(1+\alpha_{2}\right) \tau \\
& \times\left\{1+\sum_{n=1}^{+\infty} \frac{\left(\alpha_{1} \alpha_{2} k\right)^{n}\left(\gamma_{1}-\gamma_{2}\right)^{2}}{\left[\left(k^{n}-1\right) z+\gamma_{1}-k^{n} \gamma_{2}\right]^{2}}\right. \\
& \left.\quad-\alpha \sum_{n=0}^{+\infty} \frac{a_{1}^{2}\left(\alpha_{1} \alpha_{2} k\right)^{n}\left(\gamma_{1}-\gamma_{2}\right)^{2}}{\left\{\left[-\gamma_{2}+k^{n} \gamma_{1}-z_{1}\left(k^{n}-1\right)\right] z+\gamma_{1} \gamma_{2}\left(1-k^{n}\right)-z_{1}\left(\gamma_{1}-\gamma_{2} k^{n}\right)\right\}^{2}}\right\}
\end{aligned}
$$

when the circles do not touch each other.

This problem has been solved by Goree and Wilson [13] using the conformal mapping approach. Some aspects of their solution have been revisited by Budiansky and Carrier [14] and by Steif [15].

If the two inclusions are tangent to each other, then the solution is obtained as

in the matrix:

$$
\begin{aligned}
\phi_{M}^{\prime}(z)=\tau\{ & \sum_{n=-\infty}^{+\infty} \frac{\left(\alpha_{1} \alpha_{2}\right)^{|n|}}{(1+n l(z-\gamma))^{2}} \\
& -\alpha_{1} \sum_{n=0}^{+\infty} \frac{a_{1}^{2}\left(\alpha_{1} \alpha_{2}\right)^{n}}{\left\{\left(1-\gamma n l+z_{1} n l\right) z+n l \gamma^{2}-z_{1}(n l \gamma+1)\right\}^{2}} \\
& \left.-\alpha_{2} \sum_{n=0}^{+\infty} \frac{a_{2}^{2}\left(\alpha_{1} \alpha_{2}\right)^{n}}{\left\{\left(1+\gamma n l-z_{2} n l\right) z-n l \gamma^{2}+z_{2}(n l \gamma-1)\right\}^{2}}\right\},
\end{aligned}
$$


inside the first inclusion:

$$
\begin{aligned}
\phi_{I 1}^{\prime}(z)=\left(1+\alpha_{1}\right) \tau\{ & 1+\sum_{n=1}^{+\infty} \frac{\left(\alpha_{1} \alpha_{2}\right)^{n}}{(1+n l(z-\gamma))^{2}} \\
& \left.\quad-\alpha_{2} \sum_{n=0}^{+\infty} \frac{a_{2}^{2}\left(\alpha_{1} \alpha_{2}\right)^{n}}{\left\{\left(1+\gamma n l-z_{2} n l\right) z-n l \gamma^{2}+z_{2}(n l \gamma-1)\right\}^{2}}\right\},
\end{aligned}
$$

inside the second inclusion:

$$
\begin{aligned}
\phi_{I 2}^{\prime}(z)=\left(1+\alpha_{2}\right) \tau\{ & 1+\sum_{n=1}^{+\infty} \frac{\left(\alpha_{1} \alpha_{2}\right)^{n}}{(1-n l(z-\gamma))^{2}} \\
& \left.-\alpha_{1} \sum_{n=0}^{+\infty} \frac{a_{1}^{2}\left(\alpha_{1} \alpha_{2}\right)^{n}}{\left\{\left(1-\gamma n l+z_{1} n l\right) z+n l \gamma^{2}-z_{1}(n l \gamma+1)\right\}^{2}}\right\} .
\end{aligned}
$$

For the cases of two holes $\left(\alpha=\alpha_{1}=\alpha_{2}=-1\right)$ or two rigid inclusions $\left(\alpha=\alpha_{1}=\right.$ $\alpha_{2}=1$ ), the solution can be written in terms of a tabulated function, namely, the trigamma function $\psi^{\prime}[16]$ as

$\phi_{M}^{\prime}=\tau\left\{-1+\zeta^{2}\left[\psi^{\prime}(\zeta)+\psi^{\prime}(-\zeta)\right]-\alpha a_{1}^{2}\left(\frac{\zeta_{1}}{z-z_{1}}\right)^{2} \psi^{\prime}\left(\zeta_{1}\right)-\alpha a_{2}^{2}\left(\frac{\zeta_{2}}{z-z_{2}}\right)^{2} \psi^{\prime}\left(\zeta_{2}\right)\right\}$

where $\zeta, \zeta_{1}$, and $\zeta_{2}$ are defined as

$$
\begin{gathered}
\zeta=\frac{1}{l(z-\gamma)}, \\
\zeta_{1}=\frac{z-z_{1}}{l(z-\gamma)\left(z_{1}-\gamma\right)}, \\
\zeta_{2}=\frac{z_{2}-z}{l(z-\gamma)\left(z_{2}-\gamma\right)} .
\end{gathered}
$$

Some numerical results are presented in Figs. 4-8 (see pp. 491-495). The relevant quantities are plotted along the boundary of the first inclusion.

EXAMPLE 2. Interaction of a screw dislocation with two circular inclusions.

As our second example, we consider the interaction of a screw dislocation, placed at the origin, with two circular inclusions. In the absence of the inclusions, the complex potential of the screw dislocation is given by $\phi(z)=b \ln z$, where $b$ is a real constant.

A direct application of the formulae given in Secs. 4 and 5 yields the solution as in the matrix:

$$
\begin{aligned}
\phi_{M}^{\prime}(z)=b\{ & \frac{1}{z}+\sum_{n=1}^{+\infty}\left(\alpha_{1} \alpha_{2}\right)^{n} \frac{\left(M^{n} z\right)^{\prime}}{\left(M^{n} z\right)}+\sum_{n=1}^{+\infty}\left(\alpha_{1} \alpha_{2}\right)^{n} \frac{\left(N^{n} z\right)^{\prime}}{N^{n} z} \\
& \left.+\alpha_{1} \sum_{n=0}^{+\infty}\left(\alpha_{1} \alpha_{2}\right)^{n} \frac{\left(\overline{A_{1} N^{n} z}\right)^{\prime}}{\overline{A_{1} N^{n} z}}+\alpha_{2} \sum_{n=0}^{+\infty}\left(\alpha_{1} \alpha_{2}\right)^{n} \frac{\left(\overline{A_{2} M^{n} z}\right)^{\prime}}{\overline{A_{2} M^{n} z}}\right\},
\end{aligned}
$$




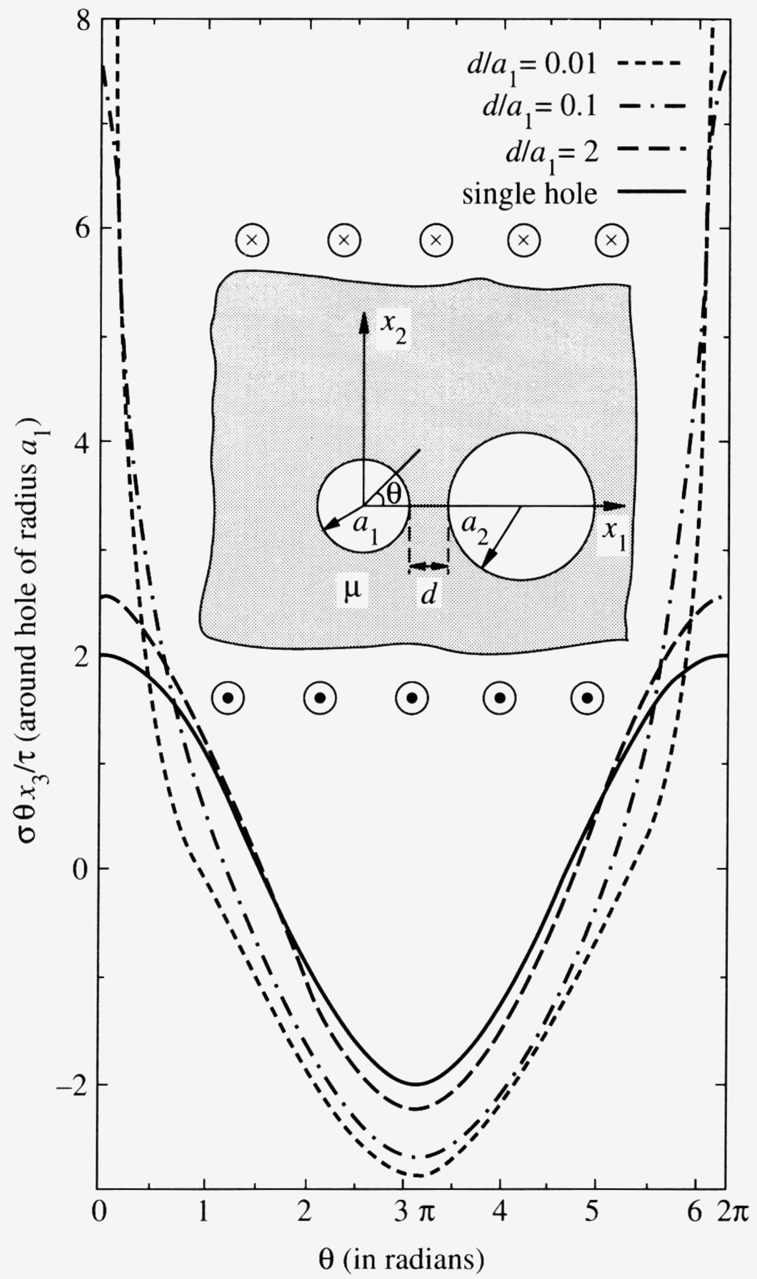

FIG. 4. Graph of $\sigma_{\theta x_{3}}(\theta)$ for the case of two holes under uniform shear:

Hole 1 centered at the origin and of radius $a_{1}$;

Hole 2 of radius $a_{2}=2 a_{1}$, centered on the real axis at $a_{1}+a_{2}+d$.

inside the first inclusion:

$$
\phi_{I 1}^{\prime}(z)=b\left(1+\alpha_{1}\right)\left\{\frac{1}{z}+\alpha_{2} \sum_{n=0}^{+\infty}\left(\alpha_{1} \alpha_{2}\right)^{n} \frac{\left(\overline{A_{2} M^{n} z}\right)^{\prime}}{\overline{A_{2} M^{n} z}}+\sum_{n=1}^{+\infty}\left(\alpha_{1} \alpha_{2}\right)^{n} \frac{\left(M^{n} z\right)^{\prime}}{M^{n} z}\right\},
$$

inside the second inclusion:

$$
\phi_{I 2}^{\prime}(z)=b\left(1+\alpha_{2}\right)\left\{\frac{1}{z}+\alpha_{1} \sum_{n=0}^{+\infty}\left(\alpha_{1} \alpha_{2}\right)^{n} \frac{\left(\overline{A_{1} N^{n} z}\right)^{\prime}}{\overline{A_{1} N^{n} z}}+\sum_{n=1}^{+\infty}\left(\alpha_{1} \alpha_{2}\right)^{n} \frac{\left(N^{n} z\right)^{\prime}}{N^{n} z}\right\},
$$

where the terms have been calculated explicitly in equations (57)-(61) and (72)-(75) when the inclusions do not touch each other. It is to be noted that the solution of this problem can be obtained by using the well-known "method of images," see for example Sendeckyj [17] who obtained the solution as a series whose general term, however, is not given explicitly but must be calculated by induction. If the two 


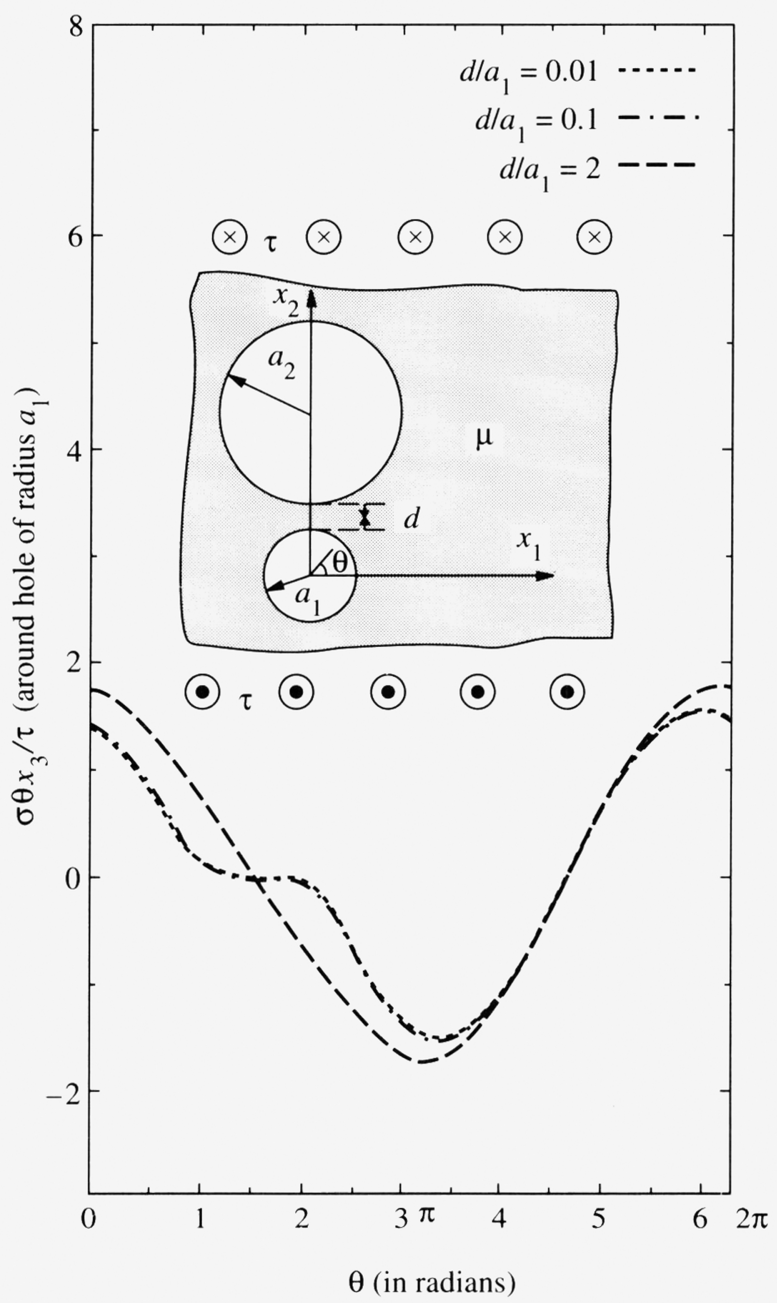

FIG. 5. Graph of $\sigma_{\theta x_{3}}(\theta)$ for the case of two holes under uniform shear: Hole 1 centered at the origin and of radius $a_{1}$; Hole 2 of radius $a_{2}=2 a_{1}$, centered on the imaginary axis at $a_{1}+a_{2}+d$.

inclusions are tangent to each other, then the expressions for the general terms are given by equations (68)-(71) and (76)-(79).

Some numerical results are presented in Figs. 9 and 10. Here also, we note that the relevant quantities are plotted along the boundary of the first inclusion.

The following observations can be made concerning Figs. 4-10.

In Fig. 4 we see that the stress concentration factor (scf), reached at $\theta=0$, becomes infinite as the two holes approach each other, while in Fig. 5, the scf for hole 1 becomes less than that of a single hole ( $\mathrm{scf}=2$ for a single hole). When the two holes approach each other, the stress on the boundary near $\theta=0$ becomes very small. This is because hole 2 acts as a shield. 


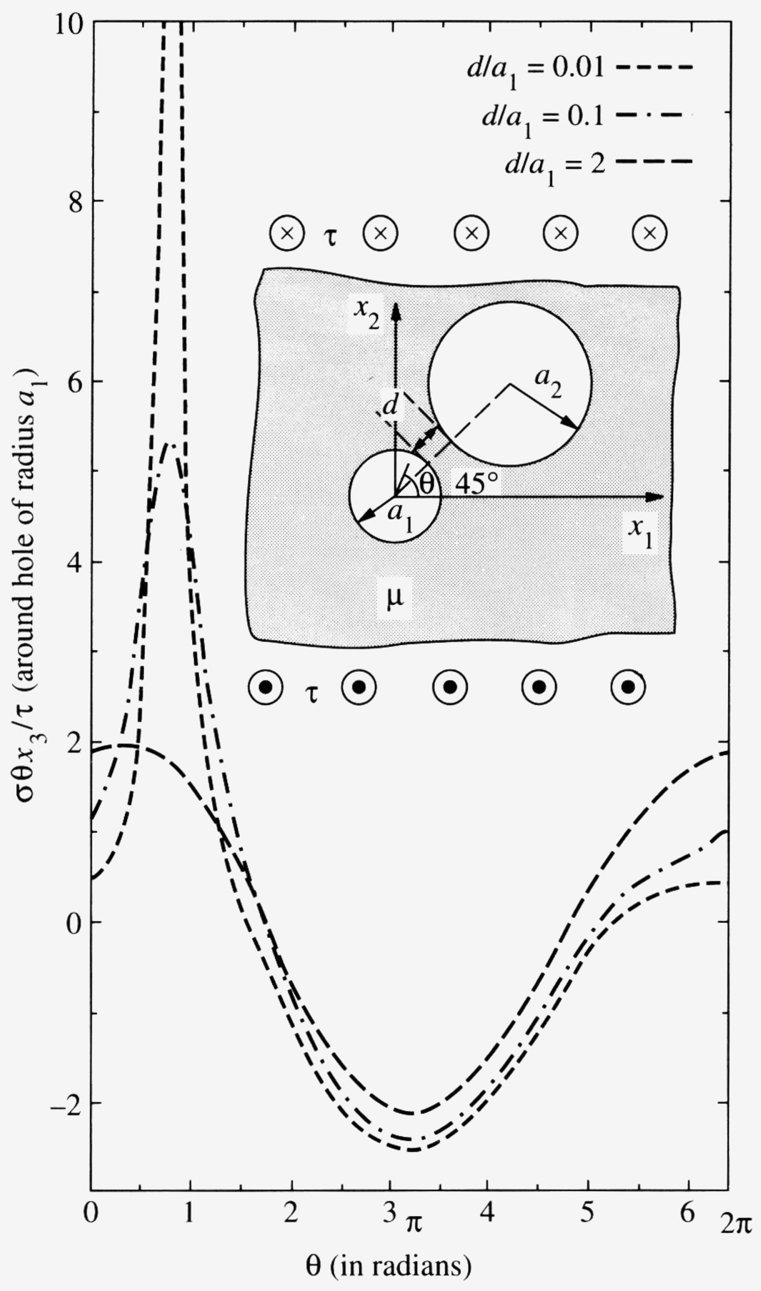

FIG. 6. Graph of $\sigma_{\theta x_{3}}(\theta)$ for the case of two holes under uniform shear: Hole 1 centered at the origin and of radius $a_{1}$; Hole 2 of radius $a_{2}=2 a_{1}$, centered in such a way that the line joining the two centers makes $45^{\circ}$ with the $x_{1}$ axis and the distance between the centers is $a_{1}+a_{2}+d$.

In Fig. 6 we can see how the maximum stress passes from 2 at $\theta=0$ to become very large at $\theta=45^{\circ}$ as the two holes approach each other on the line joining their centers.

In Fig. 7 we see that the stress is infinite at the point of tangency of two holes and is very small in its neighborhood.

The case of two holes treated in Fig. 5 is now treated in Fig. 8 for two inclusions; the case of uniform shear treated in Fig. 4 is treated for a screw dislocation in Fig. 9 on p. 496; and in Fig. 10 on pp. 496-497 we treat the case of two inclusions under a screw dislocation, as well. 


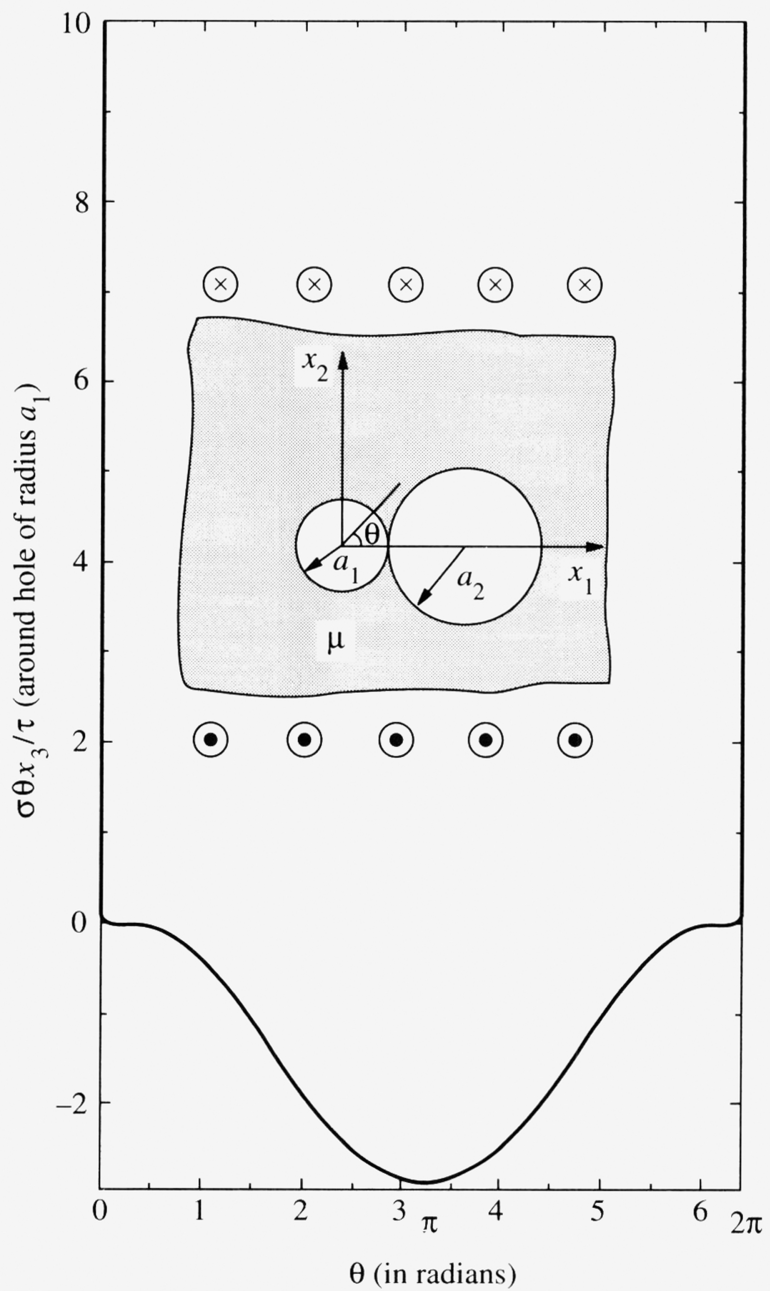

FIG. 7. Graph of $\sigma_{\theta x_{3}}(\theta)$ for the case of two holes under uniform shear and touching each other:

Hole 1 centered at the origin and of radius $a_{1}$;

Hole 2 of radius $a_{2}=2 a_{1}$, centered on the real axis at $a_{1}+a_{2}$. 


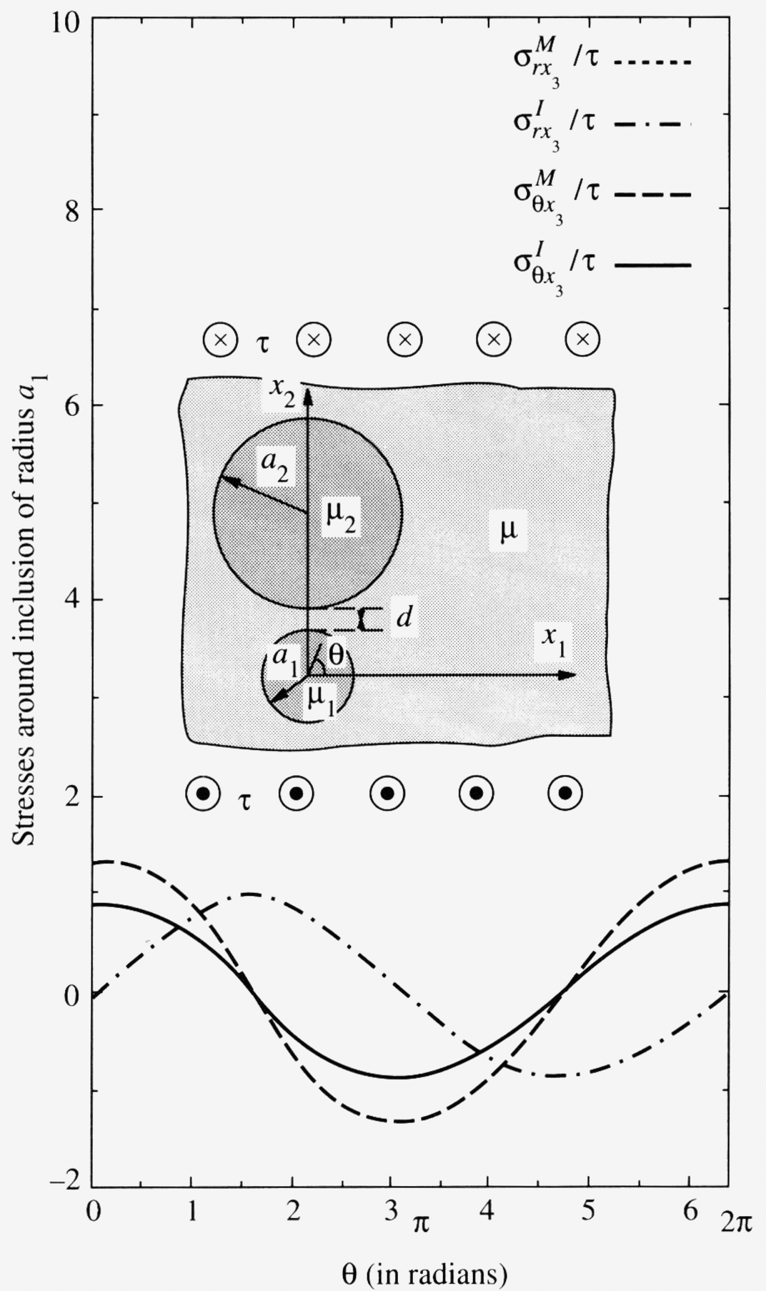

FIG. 8. Graph of $\sigma_{\theta x_{3}}(\theta)$ and $\sigma_{r x_{3}}(\theta)$ along the circular boundaries of both the matrix and the first inclusion for the case of two elastic inclusions under uniform shear:

Inclusion 1 centered at the origin and of radius $a_{1}$ and having $\alpha_{1}=-0.2$; Inclusion 2 of radius $a_{2}=2 a_{1}$, centered on the imaginary axis at $a_{1}+a_{2}+0.1 a_{1}$ $\left(d=0.1 a_{1}\right)$, and having $\alpha_{2}=0.3$.

Note that $\sigma_{r x_{3}}^{M}$ concides with $\sigma_{r x_{3}}^{I}$ (as required by the continuity of traction). 


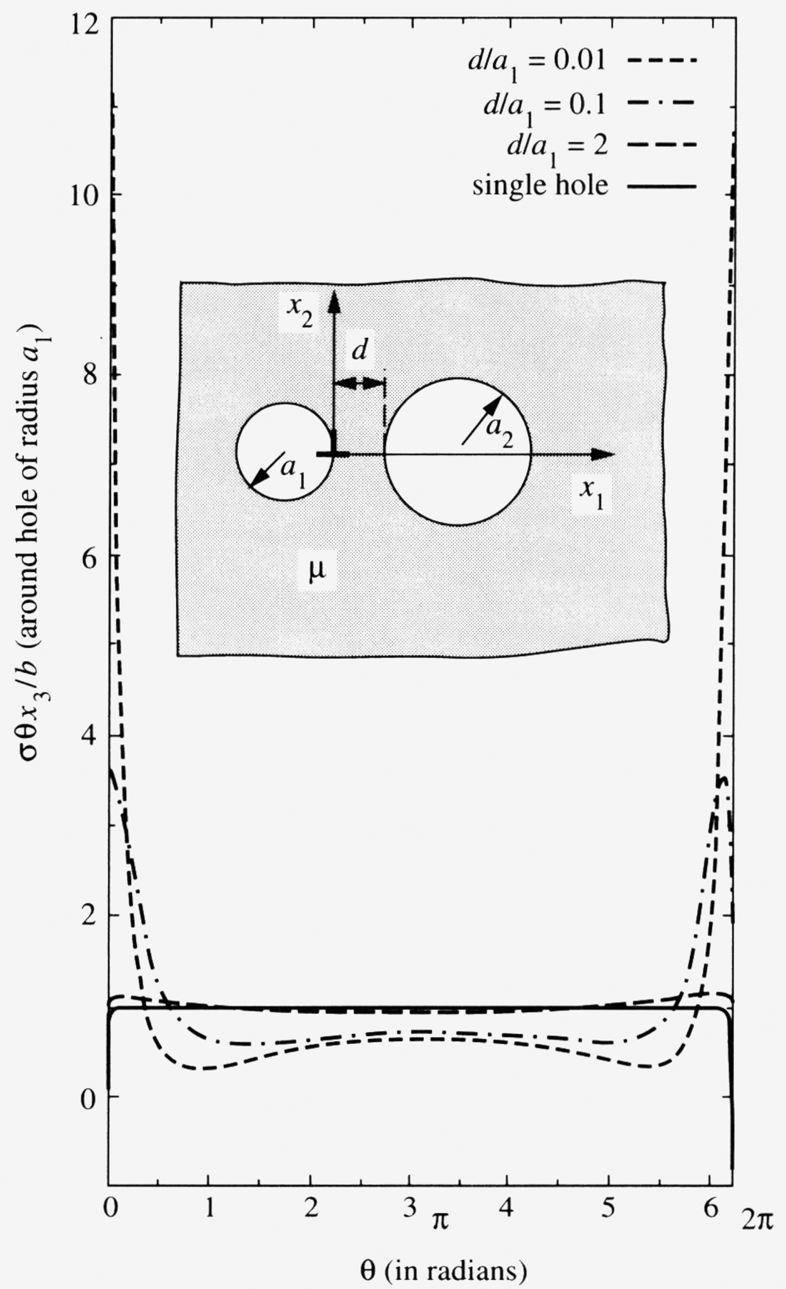

FIG. 9. Graph of $\sigma_{\theta x_{3}}(\theta)$ for the case of two holes in a screw dislocation field: The dislocation centered at the origin; Hole 1 of radius $a_{1}$, centered at $\left(-a_{1}, 0.01 a_{1}\right)$; Hole 2 of radius $a_{2}=2 a_{1}$, centered at $\left(a_{2}+d, 0.01 a_{1}\right)$. 


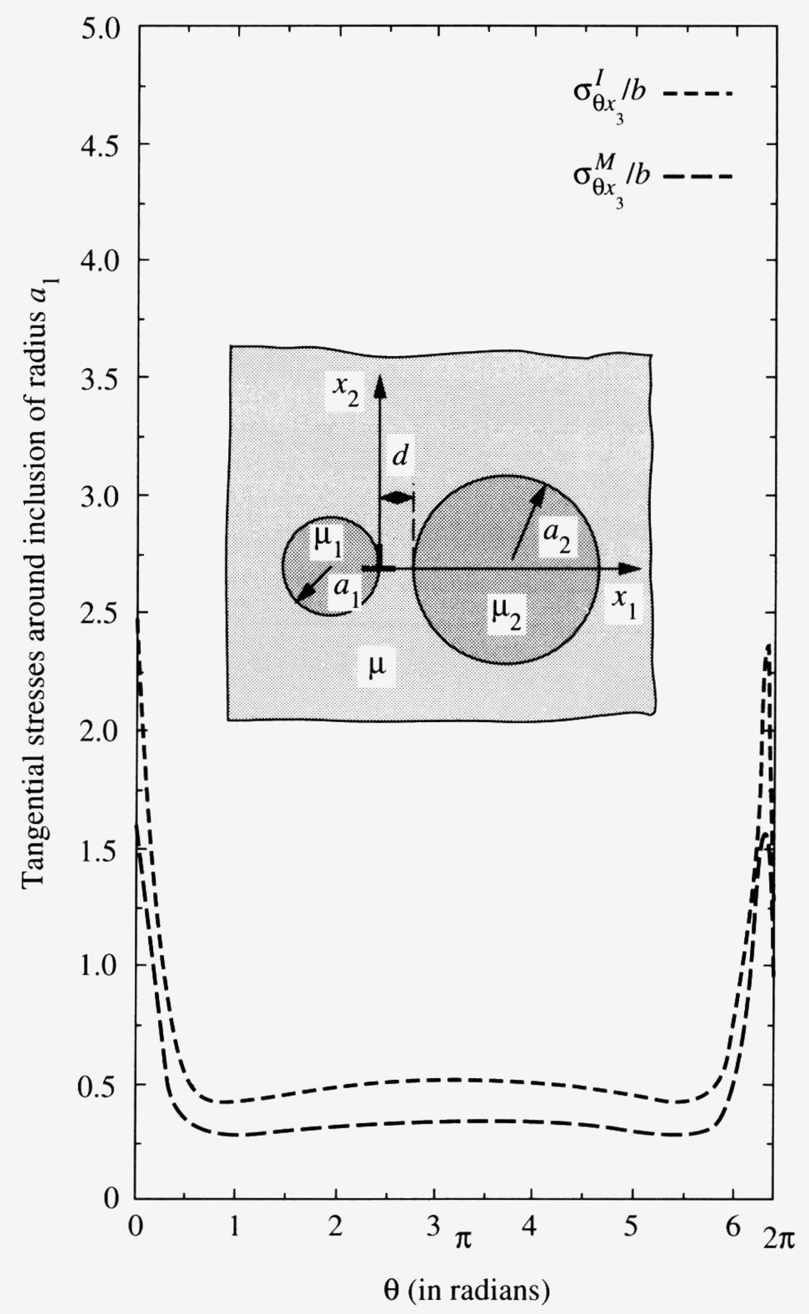

FIG. 10a. Graph of $\sigma_{\theta x_{3}}(\theta)$ along the circular boundaries of both the matrix and the first inclusion for the case of two elastic inclusions in a screw dislocation field:

The dislocation centered at the origin; The first inclusion of radius $a_{1}$, centered at $\left(-a_{1}, 0.01 a_{1}\right)$, and having $\alpha_{1}=0.2$; Inclusion 2 of radius $a_{2}=2 a_{1}$, centered at $\left(a_{2}+0.1 a_{1}, 0.01 a_{1}\right) \quad\left(d=0.1 a_{1}\right)$ and having $\alpha_{2}=-0.3$. 


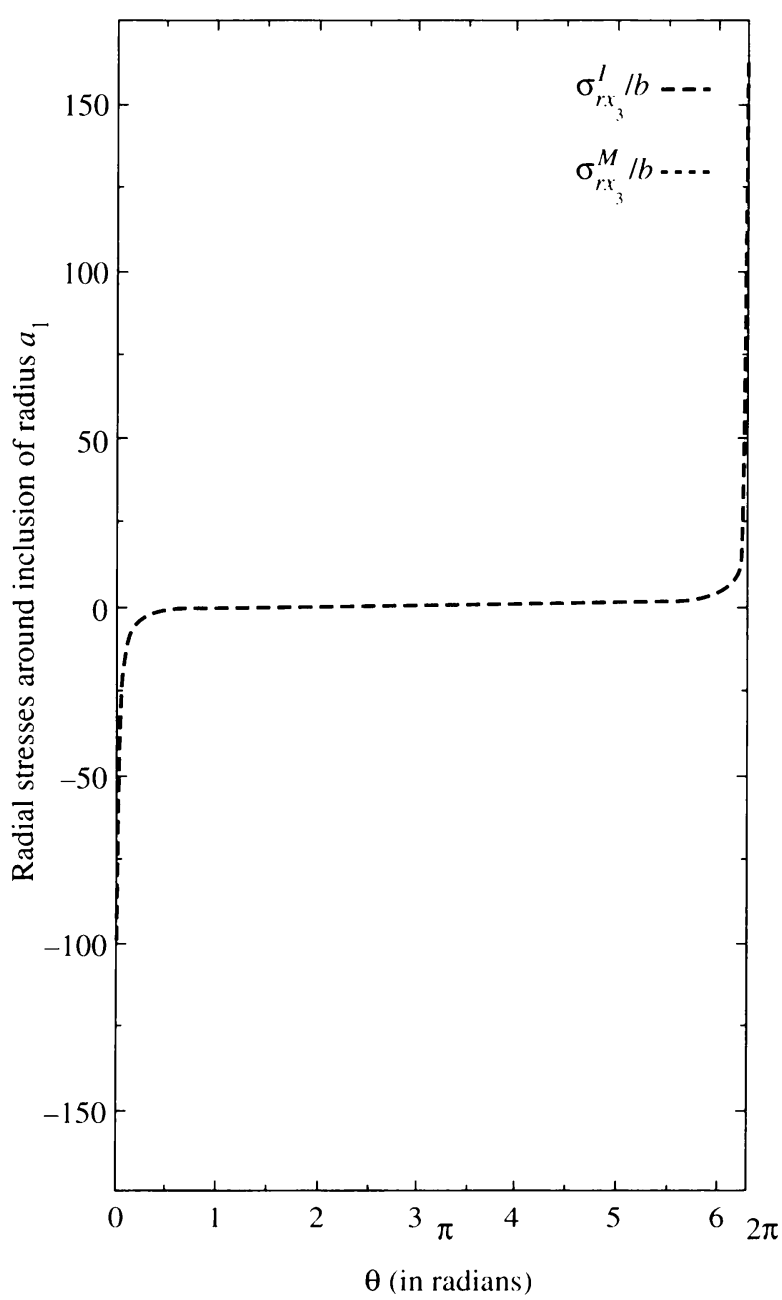

FIG. 10b. Graph of $\sigma_{r x_{3}}(\theta)$ along the circular boundaries of both the matrix and the first inclusion for the case of two elastic inclusions in a screw dislocation field. Note that $\sigma_{r x_{3}}^{I}$ coincides with $\sigma_{\sigma x_{3}}^{M}$.

7. Concluding remarks. There is a close connection between the solution given in equations (40)-(42) and the theory of discontinuous groups and automorphic functions [12]. To see this connection, we consider the case of rigid inclusions or circular holes $\left(\alpha=\alpha_{1}=\alpha_{2}= \pm 1\right)$. Then the complex potential $\phi_{M}$ given in equation (40) satisfies

$$
\phi_{M}(z)=\alpha \overline{\phi_{M}\left(A_{1} z\right)}=\alpha \overline{\phi_{M}\left(A_{2} z\right)},
$$

which implies that

$$
\phi_{M}(z)=\phi_{M}(M z),
$$

showing that $\phi_{M}$ is invariant under the action of the discontinuous group generated by $M=A_{1} A_{2}$. And therefore $\phi_{M}$ is an automorphic function (see [12]).

Once the solution for two circular inclusions is obtained, a repeated application of the above procedure leads to the solution for three circular inclusions, etc. Again 
here the theory of automorphic functions should play a crucial role. A related study has been undertaken by Burnside [18,19] and recently by Drotz [20] to determine the two-dimensional potential flow around rigid circular cylinders.

Based on this solution, the study of the energetics of the two inclusions under arbitrary loading can be carried out. However this topic, as well as the asymptotic behavior of the solution as two holes or rigid inclusions approach each other, will be discussed in forthcoming papers.

Acknowledgments. This work was performed with the support of the U. S. Department of Energy and the National Science Foundation. This support is gratefully acknowledged. The authors wish to express their gratitude to Professors A. Acrivos and J. B. Keller for helpful discussions. They would like also to thank Professor I. Ryhming of the Swiss Federal Institute of Technology in Lausanne for calling their attention to the work of A. Drotz.

\section{REFERENCES}

[1] L. M. Milne-Thomson, Hydrodynamical images, Proc. Cambridge Philos. Soc. 36, 246-247 (1940)

[2] L. M. Milne-Thomson, Theoretical Hydrodynamics, Macmillan, London, 1960

[3] W. K. H. Panofsky and M. Phillips, Classical Electricity and Magnetism, 2nd ed., Addison-Wesley, London, 1962

[4] L. D. Landau, E. M. Lifshitz, and L. P. Pitaevskiī, Electrodynamics of Continuous Media, 2nd ed., Pergamon, Oxford, 1984

[5] T. Honein and G. Herrmann, The two-dimensional problem of thermo-elasticity for an infinite region bounded by a circular cavity, Thermodynamical Couplings in Solids, H. D. Bui and Q. S. Nguyen, eds., Elsevier Science Publ. B. V. (North-Holland), Amsterdam, 1987

[6] T. Honein and G. Herrmann, The involution correspondence in plane elastostatics for regions bounded by a circle, J. Appl. Mech. 55, 566-573 (1988)

[7] T. Honein and G. Herrmann, On bonded inclusions with circular or straight boundaries in plane elastostatics, J. Appl. Mech. 57, 850-856 (1990)

[8] T. Honein and G. Herrmann, A circular inclusion with slipping interface in plane elastostatics, Micromechanics and Inhomogeneity, G. J. Weng, M. Taya, and H. Abé, eds. Springer-Verlag, 1990

[9] H. Schwerdtfeger, The Geometry of Complex Numbers, Dover, New York, 1979

[10] E. Smith, The interaction between dislocations and inhomogeneities-I, Internat. J. Engrg. Sci. 6, 129-143 (1968)

[11] Lin Wei-Wen, T. Honein, and G. Herrmann, A novel method of stress analysis of elastic materials with damage zones, Yielding, Damage, and Failure of Anisotropic Solids, EGFS, J. P. Boehler, ed., 1989, Mechanical Engineering Publ., London, 1988, 609-613

[12] L. R. Ford, Automorphic Functions, McGraw-Hill, New York, 1929; 2nd ed., Chelsea, New York, 1951

[13] J. G. Goree and H. B. Wilson Jr., Transverse shear loading in an elastic matrix containing two circular cylindrical inclusions, J. Appl. Mech., June 1967, 511-513 (1967)

[14] B. Budiansky and G. F. Carrier, High shear stress in stiff-fiber composites, J. Appl. Mech. 51. 733-735 (1984)

[15] Paul S. Steif, Shear stress concentration between holes, J. Appl. Mech. 56, 719-721 (1989)

[16] M. Abramowitz and I. Stegun, eds., Handbook of Mathematical Functions, Dover, New York, 1965

[17] G. P. Sendeckyj, Screw dislocations near circular inclusions, Phys. Status Solidi 3, 529-535 (1970)

[18] W. Burnside, On functions determined from their discontinuities and a certain form of boundary condition, Proc. London Math. Soc. (3) 22, 346-358 (1891)

[19] W. Burnside, On a class of automorphic functions, Proc. London Math. Soc. (3) 23, 49-88 (1892)

[20] Alain Drotz, Détermination analytique de potentiels d'écoulements non visqueux, incompressibles et bidimensionnels autour d'obstacles circulaires, J. Theoret. Appl. Mech. 6, 23-45 (1987) 